\title{
Sex dependent risk factors for mortality after myocardial infarction: individual patient data meta-analysis
}

Hanna M van Loo ${ }^{1 *}$, Edwin R van den Heuvel ${ }^{2}$, Robert A Schoevers ${ }^{1}$, Matteo Anselmino ${ }^{3}$, Robert M Carney ${ }^{4}$, Johan Denollet ${ }^{5}$, Frank Doyle ${ }^{6}$, Kenneth E Freedland ${ }^{4}$, Sherry L Grace ${ }^{7}$, Seyed H Hosseini ${ }^{8}$, Kapil Parakh ${ }^{9}$, Louise Pilote ${ }^{10}$, Chiara Rafanelli ${ }^{11}$, Annelieke M Roest ${ }^{1}$, Hiroshi Sato ${ }^{12}$, Richard P Steeds ${ }^{13}$, Ronald C Kessler ${ }^{14}$ and Peter de Jonge ${ }^{1}$

\begin{abstract}
Background: Although a number of risk factors are known to predict mortality within the first years after myocardial infarction, little is known about interactions between risk factors, whereas these could contribute to accurate differentiation of patients with higher and lower risk for mortality. This study explored the effect of interactions of risk factors on all-cause mortality in patients with myocardial infarction based on individual patient data meta-analysis.

Methods: Prospective data for 10,512 patients hospitalized for myocardial infarction were derived from 16 observational studies (MINDMAPS). Baseline measures included a broad set of risk factors for mortality such as age, sex, heart failure, diabetes, depression, and smoking. All two-way and three-way interactions of these risk factors were included in Lasso regression analyses to predict time-to-event related all-cause mortality. The effect of selected interactions was investigated with multilevel Cox regression models.

Results: Lasso regression selected five two-way interactions, of which four included sex. The addition of these interactions to multilevel Cox models suggested differential risk patterns for males and females. Younger women (age <50) had a higher risk for all-cause mortality than men in the same age group (HR 0.7 vs. 0.4), while men had a higher risk than women if they had depression (HR 1.4 vs. 1.1) or a low left ventricular ejection fraction (HR 1.7 vs. 1.3). Predictive accuracy of the Cox model was better for men than for women (area under the curves: 0.770 vs. 0.754).

Conclusions: Interactions of well-known risk factors for all-cause mortality after myocardial infarction suggested important sex differences. This study gives rise to a further exploration of prediction models to improve risk assessment for men and women after myocardial infarction.
\end{abstract}

Keywords: All-cause mortality, Interactions, Myocardial infarction, Prediction, Risk factors, Sex

\section{Background}

About $8 \%$ of patients die within the first months after myocardial infarction, whereas approximately $30 \%$ survive more than 20 years [1,2]. Several risk assessment instruments have been developed to quantify the risk of mortality in individual patients, such as the Global Registry of Acute Coronary Events and Thrombolysis in Myocardial Infarction risk scores [3-5]. These instruments are based on a set

\footnotetext{
* Correspondence: h.van.loo@umcg.nl

'Interdisciplinary Center Psychopathology and Emotion Regulation (ICPE), Department of Psychiatry, University of Groningen, University Medical Center Groningen, Hanzeplein 1, PO box 30.001, 9700 RB Groningen, The Netherlands Full list of author information is available at the end of the article
}

of well-known risk factors for mortality such as age, heart failure, and comorbidity. Unfortunately, interactions of these risk factors are rarely investigated optimally, while these could be of relevance in accurately differentiating the high risk from the low risk patients.

Some interactions of this type have been identified, such as between sex and age and between sex and left ventricular ejection fraction (LVEF), in predicting mortality after myocardial infarction [2,6-8]. These interactions suggested that mortality after myocardial infarction is higher in young women than in young men, while poor LVEF was associated with an increased risk of death, especially in men. However, previous studies have only investigated 
interactions sporadically and did not perform systematic searches through all or many interactions in a sufficiently large dataset to estimate interaction effects reliably. To discover currently unknown interactions of interest, we systematically investigated a large set of interactions that potentially influence prediction of all-cause mortality in patients with myocardial infarction with a novel combination of statistical learning methods $[9,10]$.

\section{Methods}

\section{Data}

Data was retrieved from the MINDMAPS dataset, an individual patient data meta-analysis dataset that combines information from 16 observational studies on baseline predictors of subsequent all-cause mortality among 10,512 patients with myocardial infarction [11]. All 16 studies began with samples of patients hospitalized for myocardial infarction according to standardized diagnostic criteria, of whom survival was assessed during follow-up. The included studies were performed in nine different countries: ten studies in Europe (Ireland, Italy, The Netherlands, Sweden, United Kingdom), four in North America (Canada, United States of America), one in Asia (Japan), and one in the Middle East (Iran). For a detailed description of the review process and study characteristics, we refer to Meijer et al. [11]. All individual studies obtained ethical approval and participants provided written informed consent [11].

\section{Measures \\ Predictors}

The predictors included those considered in previous studies of risk factors for mortality after myocardial infarction: severity of the heart disease, general health, comorbidity, sex, and age $[3,5,11]$. Severity of heart disease was indicated by history of myocardial infarction, LVEF, clinical signs of heart failure (Killip class), and betablocker use. Beta-blocker use was selected as a measure of cardiovascular medication use because information on this drug was most often available. History of myocardial infarction and beta-blocker use were dichotomized into 'yes' and 'no'; LVEF was dichotomized into significant left ventricular dysfunction present $(<40 \%)$ and absent $(\geq 40 \%)$; Killip class was dichotomized into no clinical signs of heart failure (class I) and clinical signs of heart failure (class II, III, IV) as the four-category scores were not available in all studies. Measures of general health were smoking, hyperlipidaemia, and body mass index (BMI). Smoking was dichotomized into ever (current or previous) versus never smoking. BMI was categorized into three classes: low (BMI <20), intermediate (BMI 20-30), and high (BMI >30). Comorbid diabetes mellitus, depression, and use of antidepressants were also included as predictors. The latter variables were included because of evidence from previous meta-analyses that depression independently predicts all-cause mortality in patients with coronary disease and myocardial infarction [11,12]. The level of depression was measured as a total score on either self-report questionnaires (mostly the Beck Depression Inventory (BDI), or the Zung Self-rating Depression Scale (ZSDS)) or standardized structured diagnostic interviews (such as the Composite International Diagnostic Interview, or the Structured Clinical Interview for DSM Disorders) within three months after myocardial infarction. For a detailed description of questionnaires used in the different studies, we refer to Meijer et al. [11]. Total depression scores were standardized into $z$-scores within each study in order to account for the different questionnaires. These depression $z$-scores were categorized into three classes: low ( $z$-score in lowest quartile), intermediate ( $z$-scores in intermediate quartiles), and high ( $z$-score in highest quartile). Finally, the demographic variables sex and age were included in the set of predictors. Age was categorized into low ( $<50$ years), intermediate (50-70 years), and high ( $>70$ years).

The continuous variables depression $z$-score, BMI, and age were categorized into three levels to enable the discovery of possible non-linear associations between these predictors and all-cause mortality. Based on previous studies, we expected that patients with an extreme low or high BMI, age, and depression score could potentially have a higher risk of mortality, as opposed to patients with an average score on these variables [13] or that there could be interactions with sex for extreme values of these variables, but not for average values [6,7]. Categorization of these risk factors into a low, intermediate, and high level enabled tracing such potential non-linear relationships. The intermediate classes were taken as reference classes, as they represented the largest group of patients seen in clinical practice. All logically possible two-way and three-way interactions of the predictors were included to enable a systematic data-driven investigation of interactions.

\section{Outcome}

All-cause mortality was chosen as primary outcome; time-to-event data were used.

\section{Imputation}

The extent of missing data varied across studies and some variables were not assessed in some studies. On average, $18.0 \%$ of values were missing. After pooling all studies, we imputed those values in one data set using the Multivariate Imputation by Chained Equations algorithm in $\mathrm{R}$ with predictive mean matching for continuous variables and logistic regression for binary variables (20 iterations, $R$ package mice, version 2.21) [14]. To account for possible systematic differences in the individual studies, we included 
the variable 'study' as a factor in the imputation. Survival data of cardiovascular events were included as extra predictors in the imputation to improve the imputation results, in addition to survival data of all-cause mortality and all predictors mentioned above [15].

\section{Statistical analyses}

Data were randomly divided into an $80 \%$ training sample $(\mathrm{n}=8,410)$ and a $20 \%$ validation sample $(\mathrm{n}=2,102)$. The training sample was used for model discovery with 10fold cross-validation, and the independent validation sample was used to assess the predictive performance of the models. All analyses were performed in R [16].

\section{Lasso regression analyses to identify interactions}

Least Absolute Shrinkage and Selection Operator (Lasso)penalized Cox regression was used to trace two-way and three-way interactions in addition to main effects. Penalized linear regression methods include a penalty for model complexity such as the Lasso [9]. This penalty constrains the sum of the absolute values of the regression coefficients, consequently shrinking the regression coefficients and selecting a limited set of predictors. The method is developed to increase prediction accuracy by diminishing variance or overfitting (which occurs when the model follows noise in the data too closely) in situations with many predictors. These features make Lasso regression well-suited for a situation like the current one in which a large set of interactions is analyzed and not all of them will be related to the outcome [10]. The method has been used before in other studies to select interactions in high dimensional analyses of genome-wide association studies [17].

We used the Lasso implementation for Cox regression glmnet in R, which returns a range of more to less extensive regression models dependent on the size of the Lasso penalty (version 1.9-5) $[18,19]$. Predictors included all main effects and 518 two-way and three-way interactions. Standardization of coefficients was not needed as all predictors were binary (dummy) variables. We selected the Lasso model that resulted in minimal prediction error in 10 -fold cross-validation in the $80 \%$ training sample $(n=8,410)$. Prediction error for Cox Lasso models was measured in terms of partial likelihood deviance [19]. From the Lasso model with least prediction error, we selected interactions with penalized beta coefficients $\geq 0.1$ or $\leq-0.1$ to retain the most relevant parameters. Consistency of the Lasso results was checked by repeating the same procedure 100 times in $80 \%$ random draws of the training sample $(n=6,728)$. The number of times that specific interactions were selected in those 100 Lasso regression models with beta-coefficients $\geq 0.1$ or $\leq-0.1$ was assessed and compared to the model on the full training sample $(\mathrm{n}=8,410)$.

\section{Multilevel Cox models}

Multivariate multilevel Cox regression analyses were used to estimate the unpenalized effect sizes of risk factors and interactions (R-package coxme, version 2.2-3, Breslow ties) [20]. The proportional hazards assumption for Cox regression was met [11]. Similar to the study of Meijer et al. [11], we included the individual studies as separate levels by adding variable 'study' as a random effect in the models. This was done to account for systematic differences between studies and thus the expectation that different effect sizes could be underlying the different studies $[21,22]$. The multilevel Cox models were built with data of the training sample $(n=8,410)$. First, we studied the model with main effects only. Subsequently, we checked the statistical significance of the interactions selected by the Lasso by including each to the main effects model one at a time. Stratified analyses were performed to estimate differences of effect sizes of risk factors for different subgroups of the sample in case of significant interactions.

\section{Test model performance in independent validation sample}

To account for overfitting, the predictive performance of the Cox regression model was determined in the independent $20 \%$ validation sample $(n=2,102)$ and compared to the performance in the $80 \%$ training sample $(n=8,410)$. Prediction accuracy was measured by assessing the areas under the receiving operating characteristic-curve (AUC) for predicting mortality at 3 years after myocardial infarction ( $\mathrm{R}$ package survivalROC, version 1.0-3, Kaplan Meier method) [23]. Bootstrapping was used to calculate confidence intervals of the AUC's ( $\mathrm{R}$ package boot, version 1.3$13,1,000$ bootstrapped samples, percentile bootstrap) [24].

\section{Results}

\section{Patient characteristics}

Individual patient data from each study were combined, resulting in a sample of 10,512 patients with myocardial infarction. Since we used imputation in the current study, we included 337 subjects, which is more than in the study of Meijer et al. [11]. The mean age of the sample was 61 (SD 11.9 years), $71 \%$ of the patients were male, and the mean BMI was $27 \mathrm{~kg} / \mathrm{m}^{2}$ (Table 1). Concerning the cardiac disease severity at baseline: $19 \%$ of the patients had a history of myocardial infarction prior to the index episode, $18 \%$ showed clinical signs of heart failure (Killip class II-IV), and $23 \%$ had a low LVEF $(<40 \%)$. On average, $21 \%$ of the patients had comorbid diabetes, and $40 \%$ had an elevated depression score as assessed by structured diagnostic interviews and standard cut-off values on self-report questionnaires (such as a BDI-1A $\geq 10$, or a ZSDS $\geq 40$; for a full overview see Meijer et al. [11]). Patients with elevated depression scores might be overrepresented as some studies have oversampled patients with depression [11]. Subjects in the highest quartile of 
Table 1 Baseline characteristics

\begin{tabular}{|c|c|}
\hline & Mean (s.d.)/\%* \\
\hline \multicolumn{2}{|l|}{ Demographics } \\
\hline Age (years) & $61.0(11.9)$ \\
\hline Male & 70.9 \\
\hline \multicolumn{2}{|l|}{ Heart disease } \\
\hline History of Ml & 18.8 \\
\hline LVEF $<40 \%$ & 23.0 \\
\hline Killip class II-IV & 18.1 \\
\hline Beta-blocker use & 72.0 \\
\hline \multicolumn{2}{|l|}{ Comorbidity } \\
\hline Diabetes & 21.3 \\
\hline Elevated depression score ${ }^{\dagger}$ & 39.7 \\
\hline $\mathrm{BDI}$ in highest quartile depression $z$-score ${ }^{\ddagger}$ & $21.0(7.7)$ \\
\hline $\mathrm{BDI}$ in intermediate quartiles depression $z$-score ${ }^{\ddagger}$ & $8.8(4.3)$ \\
\hline $\mathrm{BDI}$ in lowest quartile depression $z$-score ${ }^{\ddagger}$ & $2.3(2.1)$ \\
\hline Antidepressant use & 6.5 \\
\hline \multicolumn{2}{|l|}{ General health } \\
\hline BMI $\left(\mathrm{kg} / \mathrm{m}^{2}\right)$ & $27.0(5.0)$ \\
\hline Smoking (ever) & 44.7 \\
\hline Hyperlipidaemia & 46.9 \\
\hline
\end{tabular}

BDI, Beck Depression Inventory version; BMI, Body mass index; LVEF, Left ventricular ejection fraction; MI, Myocardial infarction.

*Means of age, BDI-scores and BMI, percentages otherwise of original data. Differences in baseline characteristics with respect to Meijer et al. [11] are due to the fact we included 377 patients more as we used imputation of missing values for depression.

${ }^{\dagger}$ Elevated depression score as assessed by structured diagnostic interviews and standard cut-off values on self-report questionnaires.

${ }^{\ddagger}$ Mean BDI-1A scores for subjects in highest, intermediate and lowest quartile of

depression $z$-scores for $n=6,423$ subjects in which BDI-1A scores were measured.

depression $z$-scores had a mean score on the BDI-1A of 21.0 and $10.7 \%$ used antidepressants, as opposed to $4.9 \%$ in the group with lower depression $z$-scores. The mean follow-up time was 3.6 years (median 3.0 years). Maximum follow-up time varied across studies between 1.0 and 8.2 years. In total, $14 \%$ of the patients died during follow-up.

\section{Lasso regression: selection of interactions}

Eight main effects and seven two-way interactions were found to be stable predictors of mortality with penalized beta coefficients $\geq 0.1$ or $\leq-0.1$ in Lasso regression analysis of the full training sample $(n=8,410$, Table 2$)$. The main effects older age ( $>70$ years), a history of myocardial infarction, and clinical signs of heart failure (Killip class II-IV) strongly predicted all-cause mortality. Also a poor LVEF, comorbid diabetes, and a low BMI were associated with a higher risk of all-cause mortality. Instead, a younger age ( $<50$ years) and beta-blocker use were protective. Four of the selected interactions concerned sex, in combination with a younger age ( $<50$ years), high depression score, low
Table 2 Selected main effects and interactions in Lasso regression analysis

\begin{tabular}{lll}
\hline & $\boldsymbol{\beta}^{*}$ & Frequency $^{\dagger}$ \\
\hline Demographics & & \\
Age $<50$ & -0.47 & \\
Age $>70$ & 0.86 & \\
Heart disease & & \\
History of MI & 0.52 & \\
LVEF <40\% & 0.31 & \\
Killip class II-IV & 0.54 & \\
Beta-blocker use & -0.53 & \\
Comorbidity & & \\
Diabetes & 0.47 & \\
General health & & \\
BMI <20 & 0.20 & $65 / 100$ \\
Selected interactions & & $68 / 100$ \\
Killip class * beta-blocker use & 0.26 & $64 / 100$ \\
Male sex * LVEF <40\% & 0.14 & $64 / 100$ \\
Male sex * age <50 & -0.18 & $61 / 100$ \\
Male sex * depression high & 0.13 & $36 / 100$ \\
Male sex * hyperlipidaemia & -0.17 & \\
Diabetes * beta-blocker use & 0.10 & \\
Killip class * hyperlipidaemia & 0.10 & \\
\hline B, Penalzed becoefcient BMl Body mass & & \\
\hline
\end{tabular}

$\beta$, Penalized beta-coefficient; BMI, Body mass index; LVEF, Left ventricular ejection fraction; Ml, Myocardial infarction.

${ }^{*}$ All main effects and interactions with a penalized beta-coefficient $\geq 0.1$ or $\leq-0.1$ selected in Lasso regression analysis in the training data $(n=8,410)$

${ }^{\dagger}$ The number of times this interaction was found with a beta-coefficient $\geq 0.1$

or $\leq-0.1$ in 100 Lasso regression analyses in random $80 \%$ samples of the training data $(n=6,728)$.

${ }^{\ddagger}$ Depression high: depression $z$-score in highest quartile.

LVEF (<40\%), and hyperlipidaemia. Two of the selected interactions were a combination of beta-blocker use and clinical signs of heart failure (Killip class II-IV) or diabetes. The last interaction consisted of a combination of heart failure and hyperlipidaemia. No three-way interactions had sufficiently large effect sizes to be selected.

Five of the selected interactions were observed $>50$ times in the 100 Lasso analyses on different $80 \%$ random subsamples of the training data which were performed to check the consistency of the results. These five concerned the four interactions including sex and the interaction of beta-blocker use and heart failure (Killip class II-IV). No other interactions were selected more than 50 times in the 100 Lasso runs (Additional file 1: Table S1). The five interactions that were observed more than 50 times were selected for inclusion in the multilevel Cox regression models.

\section{Cox regression: effect of interactions}

Nearly all predictors were significantly associated with all-cause mortality in the unstratified multilevel Cox 
model with main effects only ( $P$ values $<0.05$; Table 3 ). Risk factors with the largest effect sizes were an older age (>70 years), diabetes, and all measures of heart disease: clinical signs of heart failure, history of myocardial infarction, and a low LVEF (hazard ratio (HR) range 1.6 to 2.8 ). Less strong, but also significantly predicting all-cause mortality, were a low BMI $\left(<20 \mathrm{~kg} / \mathrm{m}^{2}\right)$, high depression score, and use of antidepressants (all HRs of 1.3). Significant protective factors were a younger age ( $<50$ years) and beta-blocker use (HR's of 0.5 and 0.8 , respectively). Further, a depression score in the lowest quartile predicted lower mortality (HR 0.9), which indicated that depression scores were linearly associated with mortality, as opposed to predicting mortality only from a certain cut-off. Not significantly related to all-cause mortality were sex, smoking, a high BMI $\left(>30 \mathrm{~kg} / \mathrm{m}^{2}\right)$, and hyperlipidaemia. The predictive accuracy of the model in

Table 3 Effect sizes of risk factors in unstratified multilevel Cox model

\begin{tabular}{|c|c|c|c|}
\hline & $\mathrm{HR}^{*}$ & $95 \% \mathrm{Cl}$ & $P$ \\
\hline \multicolumn{4}{|l|}{ Demographics } \\
\hline Male sex & 1.0 & $(0.9-1.1)$ & 0.86 \\
\hline Age $<50$ & 0.5 & $(0.4-0.6)$ & $<0.001$ \\
\hline Age $>70$ & 2.7 & $(2.4-3.1)$ & $<0.001$ \\
\hline \multicolumn{4}{|l|}{ Heart disease } \\
\hline History of Ml & 1.8 & $(1.5-2.0)$ & $<0.001$ \\
\hline LVEF $<40 \%$ & 1.6 & $(1.4-1.8)$ & $<0.001$ \\
\hline Killip class II-IV & 2.1 & $(1.8-2.3)$ & $<0.001$ \\
\hline Beta-blocker use & 0.8 & $(0.7-0.9)$ & $<0.001$ \\
\hline \multicolumn{4}{|l|}{ Comorbidity } \\
\hline Diabetes & 1.8 & $(1.6-2.0)$ & $<0.001$ \\
\hline Depression low ${ }^{\dagger}$ & 0.9 & $(0.7-1.0)$ & 0.04 \\
\hline Depression high $^{\dagger}$ & 1.3 & $(1.1-1.4)$ & $<0.001$ \\
\hline Antidepressant use & 1.3 & $(1.1-1.6)$ & 0.01 \\
\hline \multicolumn{4}{|l|}{ General health } \\
\hline Hyperlipidaemia & 0.9 & $(0.8-1.0)$ & 0.08 \\
\hline Smoking & 1.1 & $(1.0-1.2)$ & 0.22 \\
\hline $\mathrm{BMI}<20$ & 1.3 & $(1.0-1.7)$ & 0.03 \\
\hline $\mathrm{BMI}>30$ & 0.9 & $(0.8-1.0)$ & 0.09 \\
\hline \multicolumn{4}{|l|}{ Predictive accuracy } \\
\hline AUC (general) $)^{\ddagger}$ & \multicolumn{3}{|c|}{$0.770(0.730-0.809)$} \\
\hline AUC (male - female) $)^{\S}$ & \multicolumn{3}{|c|}{$0.770(0.714-0.825)-0.754(0.687-0.816)$} \\
\hline
\end{tabular}

AUC, Area under the curve; BMI, Body mass index; $95 \% \mathrm{Cl}, 95 \%$ Confidence interval; HR, Hazard ratio; LVEF, Left ventricular ejection fraction; MI, Myocardial infarction; $P, P$ value.

${ }^{*} \mathrm{HR}$ 's, $95 \% \mathrm{Cl}, P$ values and predictive accuracy for multivariate multilevel Cox models with main effects only in the training data $(n=8,410)$.

${ }^{\dagger}$ Depression low and high: depression $z$-score in the lowest and highest quartile.

${ }^{\ddagger} A \cup C$ in the validation data $(n=2,102)$.

${ }^{\S}$ AUC for male patients $(n=1,475)$ and female patients $(n=627)$ in the validation data. the validation sample was fair (AUC 0.770). As expected, the model was more accurate in the training sample (AUC 0.812). Because the selected interactions mainly indicated sex differences, we tested the predictive accuracy of the model for men and women separately in post-hoc analysis of the validation data. The model was more accurate for men (AUC 0.770) than for women (AUC 0.754).

Evaluation of the five selected interactions with multilevel Cox model showed that some risk factors had different effect sizes in specific subgroups of patients with myocardial infarction and indicated sex differences in particular. Four out of five Lasso-selected interactions were significant or borderline significant when they were added to the unstratified multilevel Cox model one at a time ( $P$ values 0.02 to 0.08 ; Table 4 ). The only selected, non-significant interaction was sex in combination with hyperlipidaemia. The remaining sex-related two-way interactions, which were not selected by Lasso, were also not significant ( $P$ values 0.17 to 0.99 ). With sex-stratified analysis, we investigated the differential effect sizes of risk factors for men and women as suggested by the sex-related interactions. The interaction between sex and age $<50$ years suggested that, for women, a young age was less protective than for men (HR of 0.7 vs. 0.4, respectively, as compared to the reference class of 50 to 70 years; Additional file 2: Figure S1). Second, the interaction between sex and LVEF $<40 \%$ suggested that a low LVEF was a stronger predictor of all-cause mortality in men (HR 1.7) than in women (HR 1.3; Additional file 3: Figure S2). Third, the interaction between sex and high depression score suggested that a high depression score increased the risk of all-cause mortality in men (HR 1.4), while it did not for women (HR 1.1; Additional file 4: Figure S4). Thus, young age, poor LVEF, and high depression had different effect sizes for men and women, but the direction of the effects were similar in both sexes (as opposed to increasing risk for men and decreasing risk for women or vice versa). A second stratified analysis was performed to investigate the interaction most often selected by Lasso, which was between beta-blocker use and clinical signs of heart failure (Killip class II-IV). This interaction was significant and suggested that, for patients without heart failure, beta-blocker use was more protective than for patients with heart failure (HR's of 0.7 and 0.9 , respectively, $P$ value 0.016 ; Additional file 5 : Figure S4).

\section{Incidence rates}

As most interactions involved sex, we calculated, in posthoc analysis, the incidence rates of all-cause mortality in the 3 years after myocardial infarction among men and women in relation to age, depression score, and LVEF in the entire dataset $(n=10,512)$. In general, women had a higher risk of dying than men (3-year incidence rates of 
Table 4 Sex-stratified multilevel Cox models

\begin{tabular}{|c|c|c|c|c|c|c|c|}
\hline & \multicolumn{3}{|c|}{ Female } & \multicolumn{3}{|c|}{ Male } & \multirow{2}{*}{$\begin{array}{l}\text { Inter } \\
P^{+}\end{array}$} \\
\hline & $\mathrm{HR}^{*}$ & $95 \% \mathrm{Cl}$ & $P$ & $\mathrm{HR}^{*}$ & $95 \% \mathrm{Cl}$ & $P$ & \\
\hline \multicolumn{8}{|l|}{ Demographics } \\
\hline Age $<50$ & 0.7 & $(0.4-1.1)$ & 0.087 & 0.4 & $(0.3-0.6)$ & $<0.001$ & $0.076^{\ddagger}$ \\
\hline Age $>70$ & 2.5 & $(2.0-3.1)$ & $<0.001$ & 2.9 & $(2.5-3.3)$ & $<0.001$ & 0.17 \\
\hline \multicolumn{8}{|l|}{ Heart disease } \\
\hline History of Ml & 1.8 & $(1.4-2.3)$ & $<0.001$ & 1.7 & $(1.5-2.0)$ & $<0.001$ & 0.99 \\
\hline LVEF $<40 \%$ & 1.3 & $(1.1-1.6)$ & 0.018 & 1.7 & $(1.5-2.0)$ & $<0.001$ & $0.018^{\ddagger}$ \\
\hline Killip class II-IV & 2.1 & $(1.7-2.6)$ & $<0.001$ & 2.1 & $(1.8-2.4)$ & $<0.001$ & 0.41 \\
\hline Beta-blocker use & 0.7 & $(0.6-0.9)$ & 0.001 & 0.8 & $(0.7-0.9)$ & 0.001 & 0.72 \\
\hline \multicolumn{8}{|l|}{ Comorbidity } \\
\hline Diabetes & 1.8 & $(1.4-2.2)$ & $<0.001$ & 1.7 & $(1.5-2.0)$ & $<0.001$ & 0.89 \\
\hline Depression low & 0.8 & $(0.6-1.1)$ & 0.27 & 0.9 & $(0.7-1.0)$ & 0.13 & 0.67 \\
\hline Depression high & 1.1 & $(0.9-1.4)$ & 0.41 & 1.4 & $(1.2-1.6)$ & $<0.001$ & $0.054^{\ddagger}$ \\
\hline Antidepressant use & 1.2 & $(0.8-1.6)$ & 0.37 & 1.3 & $(1.1-1.7)$ & 0.017 & 0.36 \\
\hline \multicolumn{8}{|l|}{ General health } \\
\hline Hyperlipidaemia & 1.0 & $(0.8-1.2)$ & 1.0 & 0.8 & $(0.7-1.0)$ & 0.037 & $0.26^{\ddagger}$ \\
\hline Smoking & 0.9 & $(0.7-1.2)$ & 0.54 & 1.2 & $(1.0-1.4)$ & 0.068 & 0.48 \\
\hline $\mathrm{BMI}<20$ & 1.4 & $(1.0-2.1)$ & 0.071 & 1.3 & $(0.9-1.8)$ & 0.14 & 0.91 \\
\hline $\mathrm{BMI}>30$ & 0.9 & $(0.7-1.1)$ & 0.33 & 0.9 & $(0.7-1.1)$ & 0.19 & 0.64 \\
\hline
\end{tabular}

BMI, Body mass index; $\mathrm{Cl}$, Confidence interval; HR, Hazard ratio; Inter., Interaction; LVEF, Left ventricular ejection fraction; MI, Myocardial infarction; $P, P$ value. *HR's, Cl's and $P$ values for sex-stratified multivariate multilevel Cox models in the training data $(n=8,410)$.

${ }^{\dagger} P$ value of two-way interactions between sex and all other risk factors based on addition of the interaction to the unstratified multilevel Cox model (such as in Table 3) one at the time.

${ }^{\ddagger} P$ value of the sex-related interactions selected by Lasso.

$17.0 \%$ vs. $12.0 \%)$. Nevertheless, men were more at risk of dying than women in the presence of certain combinations of risk factors. Most notable, the 3-year incidence rate of mortality for men was $65 \%$ when they were older than 70 years and had a high depression score and a low LVEF as compared to $55 \%$ for women with the same risk profile. Differences in 3-year incidence rates of all-cause mortality were more pronounced in men ( $\min .2 \%-\max$. $65 \%$ ), than in women (min. $4 \%-\max .55 \%$; Figure 1 ).

\section{Discussion}

With a systematic data-driven search through all possible two-way and three-way interactions of risk factors for all-cause mortality after myocardial infarction, we found that some risk factors act differently in men and women. A high depression score was associated with increased mortality risk in men, but not in women. In addition, for women, a younger age was less protective than for men; LVEF $<40 \%$ was more strongly predicting mortality in men than in women. Another main finding was that, in patients without heart failure, beta-blocker use was more protective than expected.

These results are limited in several ways. First, the data came from studies carried out between 1985 and 2006. As the group of patients and treatment of myocardial infarction are changing over time (more older patients,

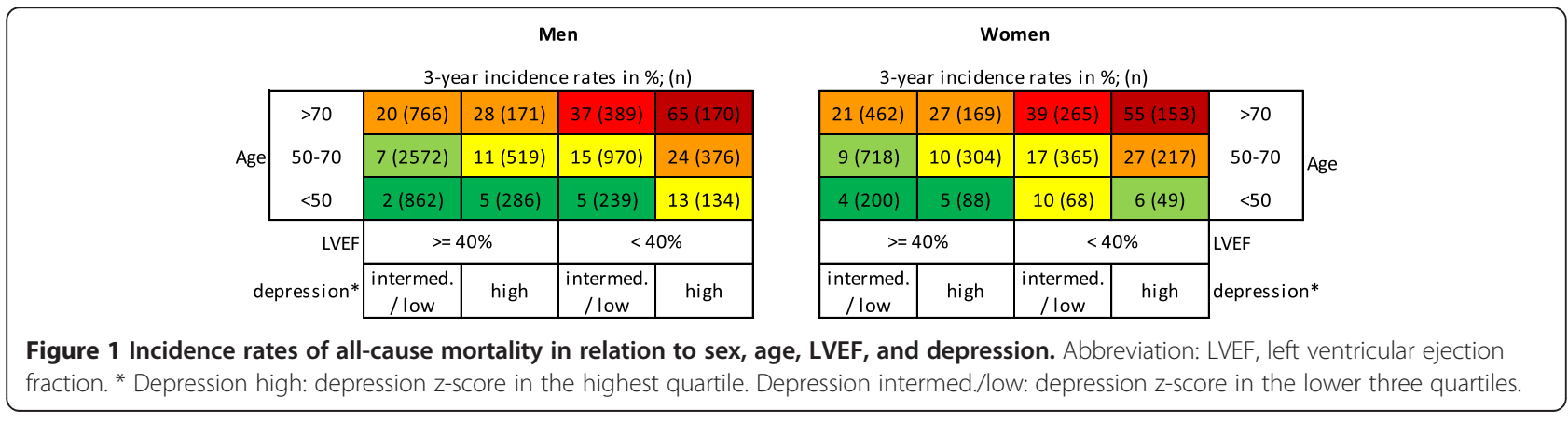


more female patients, and more comorbidity) [1,25,26], we should be cautious in generalizing the results to current patients with myocardial infarction. Indeed, replication is needed with newer data. Second, we could not include all relevant predictors, such as blood pressure, heart rate, waist circumference, kidney function, electrocardiogram findings, or educational attainment, marital status, and socioeconomic status, in our analyses as these data were not available in all studies [3-5,27,28]. Inclusion of such measures could have led to more precise risk assessment and possibly lead to other interaction patterns.

Within the context of these limitations, this is the first study performing a systematic search through a variety of interactions of risk factors for all-cause mortality after myocardial infarction using a large multi-study international sample. We applied statistical learning techniques to prevent overfitting, and tested the prediction of the identified model in an independent validation sample. Furthermore, selected interactions were clinically relevant as they indicated the importance of sex in differentiating patients with high and low risk for all-cause mortality after myocardial infarction.

Some interactions that were identified were congruent with previous studies, while others represented novel associations. Certain interactions, including sex, have been reported before such as the interaction between sex and age indicating that young women are at higher risk of all-cause mortality than young men [6,7,29]. Likewise, the interaction between sex and LVEF - suggesting that a low LVEF is a more important risk factor for men than for women - was consistent with findings in patients with myocardial infarction [8] and heart failure [30]. Two studies that focused on the interaction between sex and depression did not find substantial sex differences with regard to depression as opposed to our study, but the former studies included a relatively low number of women ( $\mathrm{n}=283$ and $\mathrm{n}=155$, respectively) and used major cardiac events or cardiac mortality as outcome measures rather than all-cause mortality $[8,31]$. Finally, it has been previously shown that beta-blocker use is more protective after myocardial infarction in the absence of clinical signs of heart failure (Killip class I) than in the presence of these signs (Killip classes II-III) [32], which is in agreement with the findings in this study.

Our study demonstrates the potential importance of interactions in risk assessment in medicine. If interaction effects are not taken into account, statistical models will return an average estimate of the effect of risk factors in the entire patient population (e.g., depression increases risk of all-cause mortality). Instead, interactions allow for possible differences within the patient population and show that a risk factor might have a different effect in the presence of another risk factor (e.g., depression increases risk of all-cause mortality in men but not in women). In our study, interactions repeatedly suggested sex differences. Although baseline differences between men and women with myocardial infarction are declining, there are still substantial dissimilarities. Men have a higher risk of myocardial infarction, but women have a higher risk of death following myocardial infarction. Women presenting with myocardial infarction are generally older, have more comorbidities such as diabetes, hypertension, and heart failure, increasing the risk of all-cause mortality as compared to men [2,33-35]. All these differences could explain why different risk factors might differentially predict all-cause mortality for men and women.

The results reported here suggest three broad classes of extensions in further research. First, follow-up studies could focus on additional interactions of interest in order to obtain more specific risk assessment for subgroups of patients with myocardial infarction. The investigated set of interactions in this study was not comprehensive. Moreover, as Lasso tends to leave out correlated interactions, there could be interactions of interest that we did not identify in this study such as diabetes and depression [36], smoking and sex [8], and LVEF and Killip class [37]. In addition, it would be worth looking at interactions including other well-known risk factors of all-cause mortality after myocardial infarction such as cardiac arrest at admission, family history, and anxiety disorders $[5,38,39]$. Second, more research is needed to identify mechanisms underlying the identified interactions. For instance, why is depression more strongly related to all-cause mortality in men than in women? A study in one of the datasets included in MINDMAPS found that part of this interaction effect can be explained by the fact that men with depression are more likely to have a poor LVEF than women with depression [40]. Depression reflected more severe heart disease in men but not in women, which partly explains why depressed men are more at risk for all-cause mortality than depressed women. Other confounders could underlie the remaining interaction effect between sex and depression found in this study after controlling for severity of heart disease. Thus, the interpretation of interactions should be done in the context of possible confounders. The finding that smoking did not have a significant main effect in this study should also be seen in this light. In addition to a broad set of important risk factors, such as history of myocardial infarction, heart failure, age, and comorbidity, smoking did not significantly predict all-cause mortality risk, but this does not imply that smoking on itself does not increase risk of all-cause mortality after myocardial infarction.

Finally, the interactions including sex lead to doubt on the performance of general risk assessment instruments for both sexes: are they equally accurate for men and women? Future studies could explore if different prediction algorithms for men and women would increase prediction 
accuracy, and if this benefit would outweigh the complexity for clinical practice of working with two different instruments instead of one.

\section{Conclusions}

Interactions might increase understanding and prediction of all-cause mortality after myocardial infarction. Our key finding was that marked sex differences exist, indicating that future research is warranted in order to improve risk assessment for both men and women with myocardial infarction.

\section{Additional files}

Additional file 1: Table S1. Selected interactions in 100 Lasso

regression analyses.

Additional file 2: Figure S1. Survival by sex and age. Survival curves of all subjects ( $n=10,512$ ) stratified by sex and age (in years) adjusted for all other risk factors. M, Male; F, Female.

Additional file 3: Figure S2. Survival by sex and left ventricular ejection fraction (LVEF). Survival curves of all subjects $(n=10,512)$ stratified by sex and LVEF adjusted for all other risk factors. M, Male; F, Female; LVEF, Left ventricular ejection fraction.

Additional file 4: Figure S3. Survival by sex and depression. Survival curves of all subjects ( $\mathrm{n}=10,512)$ stratified by sex and depression $z$-score adjusted for all other risk factors. High depression: depression z-score in highest quartile. Low-med. depression: depression z-score in lower three quartiles. M, Male; F, Female; dep, Depression z-score; med, Intermediate.

Additional file 5: Figure S4. Survival by Killip class and beta-blocker use. Survival curves of all subjects $(n=10,512)$ stratified by Killip class and beta-blocker use adjusted for all other risk factors. BB, Beta-blocker.

\section{Abbreviations}

AUC: Area under the receiving operating characteristic-curve; BDI: Beck Depression Inventory; BMI: Body mass index; HR: Hazard ratio; Lasso: Least absolute shrinkage and selection operator; LVEF: Left ventricular ejection fraction; ZSDS: Zung Self-rating Depression Scale.

\section{Competing interests}

In the past three years, Dr. Kessler has been a consultant for Hoffman-La Roche, Inc., Johnson \& Johnson Wellness and Prevention, and Sanofi-Aventis Group. Dr. Kessler has served on advisory boards for Mensante Corporation, Plus One Health Management, Lake Nona Institute, and U.S. Preventive Medicine. Dr. Kessler owns 25\% share in DataStat, Inc.

\section{Authors' contributions}

MA, RMC, JD, FD, KEF, SLG, SHH, KP, LP, CR, AMR, HS, RPS, and PdJ were involved in data acquisition. HMvL, PdJ, ERvdH, and RAS designed the study. $H M v L, P d J$, and ERvdH analyzed the data and interpreted the results. RCK contributed to statistical methods. HMvL and PdJ drafted the manuscript. All authors read and revised the manuscript critically and approved the final manuscript.

\section{Acknowledgements}

We thank Deirdre A. Lane, PhD, University of Birmingham, Centre for Cardiovascular Sciences, and Catharina Welin, RN, PhD, Institute of Health and Care Sciences, Sahlengrenska Academy, University of Gothenburg, for providing data for this individual patient data meta-analysis for the MINDMAPS collaboration. We thank Klaas J. Wardenaar, PhD, ICPE, Department of Psychiatry, University of Groningen, University Medical Center Groningen, for checking the correctness of the statistical analyses.

\section{Funding}

Kenneth E. Freedland is supported by the National Heart, Lung, and Blood

Institute (NIH), Bethesda, Maryland, USA. Seyed H. Hosseini is supported by a research deputy of Mazandaran University of Medical Sciences, Sari, Iran. Hiroshi Sato is supported by research grants-in-aid for Scientific Research from the Ministry of Education, Culture, Sports, Science and Technology (11794035) and from the Japan Society for the Promotion of Science (15590743, 21590894, 25350910). Peter de Jonge is supported by a VIDI grant from the Netherlands Research Foundation (NWO-ZonMW no: 016.086.397) and a VICI grant from the Netherlands Research Foundation (NWO-ZonMW no: 91812607).

\section{Author details}

${ }^{1}$ Interdisciplinary Center Psychopathology and Emotion Regulation (ICPE), Department of Psychiatry, University of Groningen, University Medical Center Groningen, Hanzeplein 1, PO box 30.001, 9700 RB Groningen, The Netherlands. ${ }^{2}$ Department of Mathematics and Computer Science, Eindhoven University of Technology, Den Dolech 2, 5612 AZ Eindhoven, The Netherlands. ${ }^{3}$ Division of Cardiology, Department of Medical Sciences, Città della Salute e della Scienza, University of Turin, C.so A.M. Dogliotti, 14, 10126 Turin, Italy. ${ }^{4}$ Department of Psychiatry, Washington University School of Medicine, 4320 Forest Park Avenue, St. Louis, Missouri 63108, USA. ${ }^{5}$ CoRPS-Center of Research on Psychology in Somatic diseases, Tilburg University, Warandelaan 2, 5000 LE Tilburg, The Netherlands. 'Division of Population Health Sciences (Psychology), Royal College of Surgeons in Ireland, 123 St Stephen's Green, Dublin 2, Ireland. 'Faculty of Health, York University and University Health Network, 368 Norman Bethune, 4700 Keele Street, Toronto M3J 1P3, Canada. ${ }^{8}$ Psychiatry and Behavioral Sciences Research Center, Addiction Institute, Mazandaran University of Medical Sciences, Psychosomatic department, Imam hospital, Sari, Iran. ${ }^{9}$ John Hopkins School of Medicine, John Hopkins Bloomberg School of Public Health, John Hoplins Bayview Medical Center, 4940 Eastern Avenue, Baltimore, Maryland 21224, USA. ${ }^{10}$ Division of General Internal Medicine, McGill University, McGill University Health Centre, 687 Pine Avenue West, V Building, V2.17, Montreal H3A 1A1, Canada. ${ }^{11}$ Department of Psychology, University of Bologna, Viale Berti Pichat 5, 40127 Bologna, Italy. ${ }^{12}$ School of Human Welfare Studies, Kwansei Gakuin University, 1-1-155, Uegahara, Nishinomiya, Hyogo 662-8501, Japan. ${ }^{13}$ Department of Cardiology, Queen Elizabeth Hospital, Edgbaston, Birmingham B15 2THWest Midlands, UK. ${ }^{14}$ Department of Health Care Policy, Harvard Medical School, 180 Longwood Avenue, Boston, Massachusetts 02115, USA.

Received: 12 September 2014 Accepted: 21 November 2014 Published online: 17 December 2014

\section{References}

1. Yeh RW, Sidney S, Chandra M, Sorel M, Selby JV, Go AS: Population trends in the incidence and outcomes of acute myocardial infarction. $N$ Engl J Med 2010, 362:2155-2165.

2. Nauta ST, Deckers JW, van Domburg RT, Akkerhuis KM: Sex-related trends in mortality in hospitalized men and women after myocardial infarction between 1985 and 2008: equal benefit for women and men. Circulation 2012, 126:2184-2189.

3. Antman EM, Cohen M, Bernink PJ, McCabe CH, Horacek T, Papuchis G, Mautner B, Corbalan R, Radley D, Braunwald E: The TIMI risk score for unstable angina/non-ST elevation Ml: A method for prognostication and therapeutic decision making. JAMA 2000, 284:835-842.

4. Morrow DA, Antman EM, Charlesworth A, Cairns R, Murphy SA, de Lemos JA, Giugliano RP, McCabe CH, Braunwald E: TIMI risk score for ST-elevation myocardial infarction: a convenient, bedside, clinical score for risk assessment at presentation: an intravenous nPA for treatment of infarcting myocardium early II trial substudy. Circulation 2000, 102:2031-2037.

5. Granger CB, Goldberg RJ, Dabbous O, Pieper KS, Eagle KA, Cannon CP, Van De Werf F, Avezum A, Goodman SG, Flather MD, Fox KA, Global Registry of Acute Coronary Events Investigators: Predictors of hospital mortality in the global registry of acute coronary events. Arch Intern Med 2003, 163:2345-2353.

6. Vaccarino V, Parsons L, Every NR, Barron HV, Krumholz HM: Sex-based differences in early mortality after myocardial infarction. National Registry of Myocardial Infarction 2 Participants. N Engl J Med 1999, 341:217-225.

7. Berger JS, Brown DL: Gender-age interaction in early mortality following primary angioplasty for acute myocardial infarction. Am J Cardiol 2006, 98:1140-1143.

8. Frasure-Smith N, Lesperance F, Juneau M, Talajic M, Bourassa MG: Gender, depression, and one-year prognosis after myocardial infarction. Psychosom Med 1999, 61:26-37.

9. Tibshirani R: Regression Shrinkage and Selection via the Lasso. J Royal Stat Soc Series B (Methodology) 1996, 58:267-288. 
10. James G, Witten D, Hastie T, Tibshirani R: An Introduction to Statistical Learning with Applications in R. New York: Springer; 2013.

11. Meijer A, Conradi HJ, Bos EH, Anselmino M, Carney RM, Denollet J, Doyle F, Freedland KE, Grace SL, Hosseini SH, Lane DA, Pilote L, Parakh K, Rafanelli C, Sato $H$, Steeds RP, Welin C, de Jonge P: Adjusted prognostic association of depression following myocardial infarction with mortality and cardiovascular events: individual patient data meta-analysis. $\mathrm{Br} J \mathrm{Psych}$ 2013, 203:90-102.

12. Lichtman JH, Froelicher ES, Blumenthal JA, Carney RM, Doering LV, Frasure-Smith N, Freedland KE, Jaffe AS, Leifheit-Limson EC, Sheps DS, Vaccarino V, Wulsin L, on behalf of the American Heart Association Statistics Committee of the Council on Epidemiology and Prevention and the Council on Cardiovascular and Stroke Nursing: Depression as a risk factor for poor prognosis among patients with acute coronary syndrome: systematic review and recommendations: a scientific statement from the American Heart Association. Circulation 2014, 129:1350-1369.

13. Bucholz EM, Rathore SS, Reid KJ, Jones PG, Chan PS, Rich MW, Spertus JA, Krumholz HM: Body mass index and mortality in acute myocardial infarction patients. Am J Med 2012, 125:796-803.

14. van Buuren S, Groothuis-Oudshoorn K: mice: Multivariate imputation by chained equations in R. J Stat Software 2011, 45:1-67.

15. Carpenter JR, Kenward MG: Multiple Imputation and its Application. Chichester: Wiley; 2013

16. R Core Team: R: A language and environment for statistical computing. Vienna: R Foundation for Statistical Computing; 2012.

17. Wu TT, Chen YF, Hastie T, Sobel E, Lange K: Genome-wide association analysis by lasso penalized logistic regression. Bioinformatics 2009, 25:714-721.

18. Friedman J, Hastie T, Tibshirani R: Regularization paths for generalized linear models via coordinate descent. J Stat Software 2010, 33:1-22.

19. Simon N, Friedman J, Hastie T, Tibshirani R: Regularization paths for Cox's proportional hazards model via coordinate descent. J Stat Software 2011, 39:1-13.

20. Thernau T: coxme: Mixed Effects Cox Models. R package version 2.2-3. Vienna: R Foundation for Statistical Computing; 2012

21. Cooper $H$, Patall EA: The relative benefits of meta-analysis conducted with individual participant data versus aggregated data. Psychol Methods 2009, 14:165-176

22. Borenstein M, Hadges LV, Higgins JPT, Rothstein HR: Introduction to MetaAnalysis. Chichester: Wiley; 2009.

23. Heagerty PJ, Saha-Chaudhuri P: survivalROC: Time-Dependent ROC Curve Estimation from Censored Survival Data. R package version 1.0.3. Vienna: R Foundation for Statistical Computing; 2013

24. Canty A, Ripley B: boot: Bootstrap R (S-Plus) Functions. R package version 1.3-11. Vienna: R Foundation for Statistical Computing; 2014

25. Gore MO, Patel MJ, Kosiborod M, Parsons LS, Khera A, de Lemos JA, Rogers WJ, Peterson ED, Canto JC, McGuire DK, National Registry of Myocardial Infarction Investigators: Diabetes mellitus and trends in hospital survival after myocardial infarction, 1994 to 2006: data from the national registry of myocardial infarction. Circ Cardiovasc Qual Outcomes 2012, 5:791-797.

26. Fokkema ML, James SK, Albertsson P, Akerblom A, Calais F, Eriksson P, Jensen J, Nilsson T, de Smet BJ, Sjogren I, Thorvinger B, Lagerqvist B: Population trends in percutaneous coronary intervention: 20 -year results from the SCAAR (Swedish Coronary Angiography and Angioplasty Registry). J Am Coll Cardiol 2013, 61:1222-1230.

27. Chang WC, Kaul P, Westerhout CM, Graham MM, Armstrong PW: Effects of socioeconomic status on mortality after acute myocardial infarction. Am J Med 2007, 120:33-39.

28. Zeller M, Steg PG, Ravisy J, Lorgis L, Laurent $Y$, Sicard P, Janin-Manificat L, Beer JC, Makki H, Lagrost AC, Rochette L, Cottin Y, RICO Survey Working Group: Relation between body mass index, waist circumference, and death after acute myocardial infarction. Circulation 2008, 118:482-490.

29. Vaccarino V, Horwitz RI, Meehan TP, Petrillo MK, Radford MJ, Krumholz HM: Sex differences in mortality after myocardial infarction: evidence for a sex-age interaction. Arch Intern Med 1998, 158:2054-2062.

30. Martinez-Selles M, Garcia Robles JA, Prieto L, Dominguez Munoa M, Frades E, Diaz-Castro O, Almendral J: Systolic dysfunction is a predictor of long term mortality in men but not in women with heart failure. Eur Heart $J$ 2003, 24:2046-2053.

31. Frasure-Smith $\mathrm{N}$, Lesperance $\mathrm{F}$ : Depression and anxiety as predictors of 2-year cardiac events in patients with stable coronary artery disease. Arch Gen Psychiatry 2008, 65:62-71.
32. Emery M, Lopez-Sendon J, Steg PG, Anderson FA Jr, Dabbous OH, Scheuble A, Eagle KA, GRACE Investigators: Patterns of use and potential impact of early beta-blocker therapy in non-ST-elevation myocardial infarction with and without heart failure: the Global Registry of Acute Coronary Events. Am Heart J 2006, 152:1015-1021.

33. Vaccarino V, Parsons L, Peterson ED, Rogers WJ, Kiefe Cl, Canto J: Sex differences in mortality after acute myocardial infarction: changes from 1994 to 2006. Arch Intern Med 2009, 169:1767-1774.

34. Velders MA, Boden $H$, van Boven AJ, van der Hoeven BL, Heestermans AA, Cannegieter SC, Umans VA, Jukema JW, Hofma SH, Schalij MJ: Influence of gender on ischemic times and outcomes after ST-elevation myocardial infarction. Am J Cardiol 2013, 111:312-318.

35. Poon S, Goodman SG, Yan RT, Bugiardini R, Bierman AS, Eagle KA, Johnston N, Huynh T, Grondin FR, Schenck-Gustafsson K, Yan AT: Bridging the gender gap: Insights from a contemporary analysis of sex-related differences in the treatment and outcomes of patients with acute coronary syndromes. Am Heart J 2012, 163:66-73.

36. Bot M, Pouwer F, Zuidersma M, van Melle JP, de Jonge P: Association of coexisting diabetes and depression with mortality after myocardial infarction. Diabetes Care 2012, 35:503-509.

37. Parakh K, Thombs BD, Bhat U, Fauerbach JA, Bush DE, Ziegelstein RC: Longterm significance of Killip class and left ventricular systolic dysfunction. Am J Med 2008, 121:1015-1018.

38. Leander K, Hallqvist J, Reuterwall C, Ahlbom A, de Faire U: Family history of coronary heart disease, a strong risk factor for myocardial infarction interacting with other cardiovascular risk factors: results from the Stockholm Heart Epidemiology Program (SHEEP). Epidemiology 2001, 12:215-221.

39. Roest $A M, Z$ Zuidersma $M$, de Jonge $P$ : Myocardial infarction and generalised anxiety disorder: 10-year follow-up. Br J Psychiatry 2012, 200:324-329.

40. van Melle JP, de Jonge P, Ormel J, Crijns HJ, van Veldhuisen DJ, Honig A, Schene AH, van den Berg MP, MIND-IT investigators: Relationship between left ventricular dysfunction and depression following myocardial infarction: data from the MIND-IT. Eur Heart J 2005, 26:2650-2656.

\section{Submit your next manuscript to BioMed Central and take full advantage of:}

- Convenient online submission

- Thorough peer review

- No space constraints or color figure charges

- Immediate publication on acceptance

- Inclusion in PubMed, CAS, Scopus and Google Scholar

- Research which is freely available for redistribution 